\section{D CT scan for perioperative identification of anatomical variations of lungs}

\author{
Anna Galluzzo ${ }^{*, 1}$, Federica Fatica², Filippo Caternicchia², Girolamo Geraci², \\ Giovanna Mancuso ${ }^{3}$, Francesco Raffaele ${ }^{2}$, Claudio Genova ${ }^{4}$, Gerlando Cocchiara², \\ Massimo Midiri ${ }^{3} \&$ Massimo Cajozzo ${ }^{2}$
}

\begin{abstract}
Aim: The aim of this study is to investigate anatomical lung variations and vascular patterns using volumetric 3D computed tomography $(C T)$ representations. Methods \& results: We considered 24 major thoracic surgery performed in our ward. In these, we discovered some interesting anatomical variations of the main pulmonary fissures. These findings were not visible on the plain $x$-ray or during routine examination of a preoperative CT scan. After re-examination of 3D CT scan reconstruction the anatomical variations were detected. Discussion: General thoracic surgeons must familiarize themselves with anatomical variations in lungs. 3D images may aid the general thoracic surgeon in performing safer surgeries. Conclusion: 3D CT scan should be performed before surgery if possible.
\end{abstract}

First draft submitted: 15 July 2016; Accepted for publication: 22 September 2016; Published online: 3 October 2016

Multidetector-row computed tomography (CT) is the primary tool for thoracic assessment. Variations of fissures are common in clinical practice; they have been reported up to $40 \%$ in anatomical specimen [1-3]. CT identification of lesions related to pulmonary fissures is the main tool for the localization of the lesions in anatomic lung lobes. Anatomical variations increase the risks of injuries and the potential for mistakes during lung resection [4], especially under video-assisted thoracic surgery (VATS). In the last few years, the number of VATS lobectomy procedures has grown up significantly; data confirm that VATS lobectomy compared with open surgical procedures has several advantages including better cosmetic results, less postoperative pain, shorter hospitalization and lower costs. However, according to some authors, there could be 'technical contraindications' in the execution of VATS lobectomy, forcing the surgeon to continue with open surgery during a VATS procedure. Among these 'technical contraindications' we should mention interlobar fissure absence/incompleteness, vascular anatomical variations, chest wall involvement, advanced age, emphysema, severe pleural adhesions, peribronchial and/or perivascular fibrosis. These represent extended indications/criteria for VATS's major pulmonary resections. As a matter of fact, we would like to describe the potential role of CT imaging reconstruction for well-localized pulmonary nodule through the evaluation of fissures and lung variants before surgery. It could be vital when making the decision about which kind of surgery to perform, either open surgery or VATS procedures.

IIBFM-CNR, DIBIMED, University of Palermo, Palermo, Italy

${ }^{2}$ Thoracic Surgery Department, University of Palermo, Palermo, Italy

${ }^{3}$ University of Palermo, Palermo, Italy

${ }^{4}$ Radiology Department, University of Palermo, Palermo, Italy

*Author for correspondence: Tel.: +39 091655 2339; anna.galluzzo@gmail.com

\section{KEYWORDS}

- anatomic variations $\bullet \mathrm{CT}$ reconstruction • lung surgery

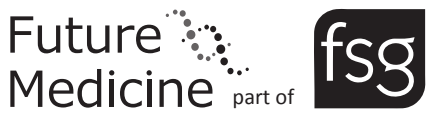




\section{Materials \& methods}

The study population consisted of 24 patients who were to undergo thoracic surgery for lung cancer. Among these, 16 patients (11 men and five women; mean age: 69 years; age range: 54-82 years) were included in the study as they had a complete set of examinations. All patients were required to provide a written informed consent before undergoing VATS or open surgery in accordance with the institutional guidelines. In this single institution, retrospective observational study, CT examinations were performed with a multidetector CT on a 16 Speedlight (GE Healthcare, WI, USA). Basic acquisition was performed from lung apices and bases at inhalation in a single breathhold. CT images were obtained using a 1-mm thick section, with a $0.625-\mathrm{mm}$ reconstruction interval between the centers of slices (overlapping slices), using 120 $\mathrm{kVp}$ and $200 \mathrm{~mA}, 512 \times 512$ matrix. Initially, CT imaging findings were retrospectively reviewed by a supervised medical student, with a possibility of consultation with experienced radiologists. Finally, in 16 patients, the images were processed, manipulated and interpreted in the workstation by two radiologists (A Galluzzo and $\mathrm{C}$ Genova) who judged whether they correctly identified the type of anatomical variations and their localization in the lungs. The picture archiving and communication systems' station was used to obtain interactive multiplanar reconstructions (MPR) and standard lung window and 3D CT representation using the workstation software tools. Data were statistically analyzed using a Fisher test. After the examination of the $3 \mathrm{D} C \mathrm{CT}$, the anatomical variations were detected. We examined the presence, extension, localization and type of incompleteness of oblique interlobar and horizontal fissures, and the presence of accessory fissures and their localization in the lungs.

\section{Results}

Radiologically, the fissures appear as bright, hypovascular band or as a sharp line [5]. Incomplete fissures have a reported incidence of $3 \%$ in 3D CT images [1]. We detected incomplete fissures in three patients and accessory fissures in two patients. The accessory fissures were located in the inferior right lobe. Vascular variations were detected at surgery, particularly, in the left lung, with fissures anomalies in the right lung. The most common variation is the socalled mediastinal lingular artery, which is the first branch of the left main pulmonary artery and was reported in $27.3 \%$ of cases [4,5]. It is difficult to identify if it is not detected preoperative, but it is clearly shown by CT representation.

Thirteen patients underwent open surgical wedge resection of the pulmonary lesion, and three patients underwent minimal invasive surgery VATS. The average age of our patients was 69 years, and all of them were out of the hospital in good clinical conditions after the shelter period. In some of these patients, we discovered interesting anatomical variations of the main fissures and vessels in both lungs during surgery. These findings were not visible on the plain $\mathrm{x}$-ray

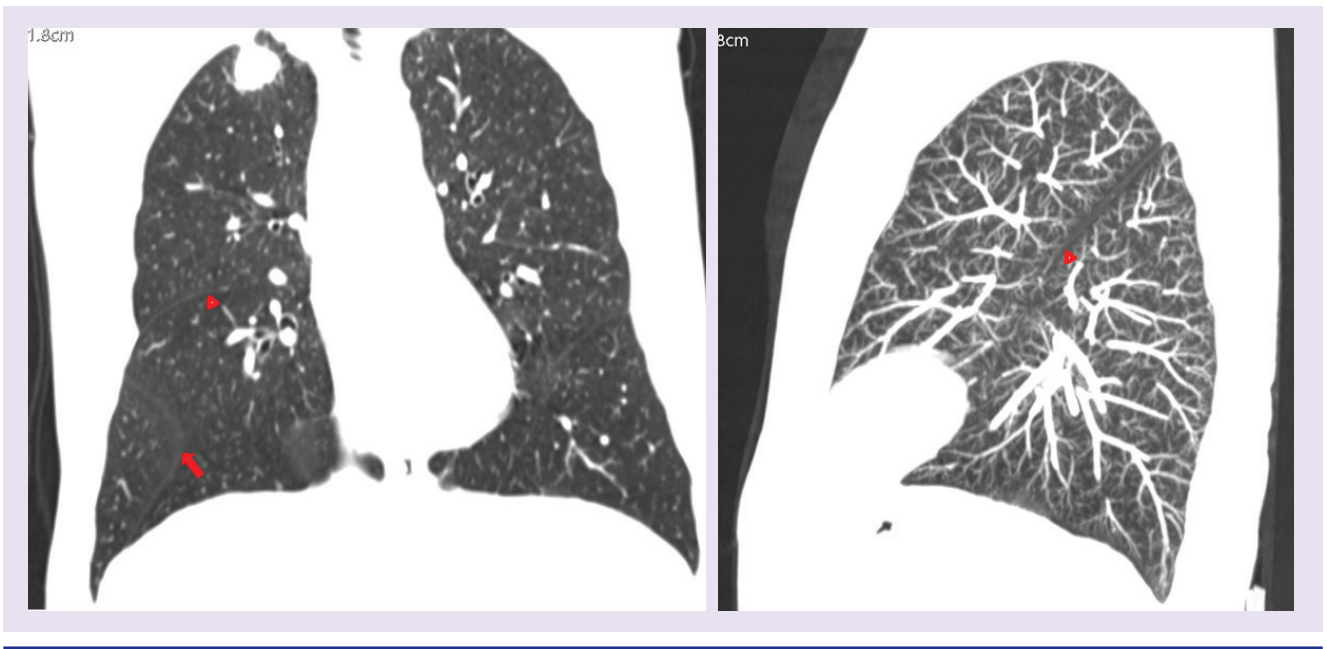

Figure 1. Coronal and sagittal maximum intensity projection reconstruction in a 72-year-old man with right apical nodule. Incomplete major fissure toward the mediastinal side (head of the arrow) and accessory fissure (arrow). 
or not signaled during routine examination of preoperative CT scan. Thus, 3D images of the pulmonary fissures and vessels were shown in 3D preoperative CT images. In a 72-year-old man, we found a correlation between the presence of incomplete and accessory fissure in the lower right lobe, visible only through CT (Figure 1). In another older male patient, the neoplastic disease involved the minor paracardiac lobar fissure and affected its imaging appearance. Atypical localization of the interlobar fissure was defined as 5\% of the least frequent combinations of localization described earlier.

\section{Discussion}

Multidetector CT is superior to conventional radiography in detecting small pulmonary lesions and to evaluate the clinical usefulness of intervention [6]. The CT images with contrast media administration were performed to evaluate vascular structures whose anatomic variants can modify treatment. The vascular structures and fissures, in particular, were visualized with maximum intensity projection, MPR and volume rendering (VR) representation. The use of postprocessing representations provides the surgeon quick, decisive and valid assistance when making a decision. Accessory fissures of the lung have common variations, often found in anatomical specimens, but are infrequently demonstrated on CT scan [7]. General thoracic surgeons must be familiar with anatomical variations in the pulmonary interlobar fissures and vessels. VATS is a very useful method to see the details of structures while performing the operation. On the other hand, it is relatively difficult to see the whole lung and the anatomy of vessels and bronchi [8].

The $3 \mathrm{D}$ representation for enormous quantity of data can be utilized in clinical practice and in surgery. The final images can reveal various lesions or organs of interest with high anatomical details and accuracy to the general thoracic surgeon, which is helpful in performing safer surgeries (Figure 2) [4,9].

Complete preoperative knowledge of the pulmonary bronchovascular pattern, including rare anatomical variations and a full understanding of individual anatomy, is imperative before performing lobectomy [9]. This knowledge leads to optimal operative assessment and strategic planning.

Recently, advances in multidetector-row CT and imaging techniques using $\mathrm{VR}$ reconstruction software have allowed 3D image representations.

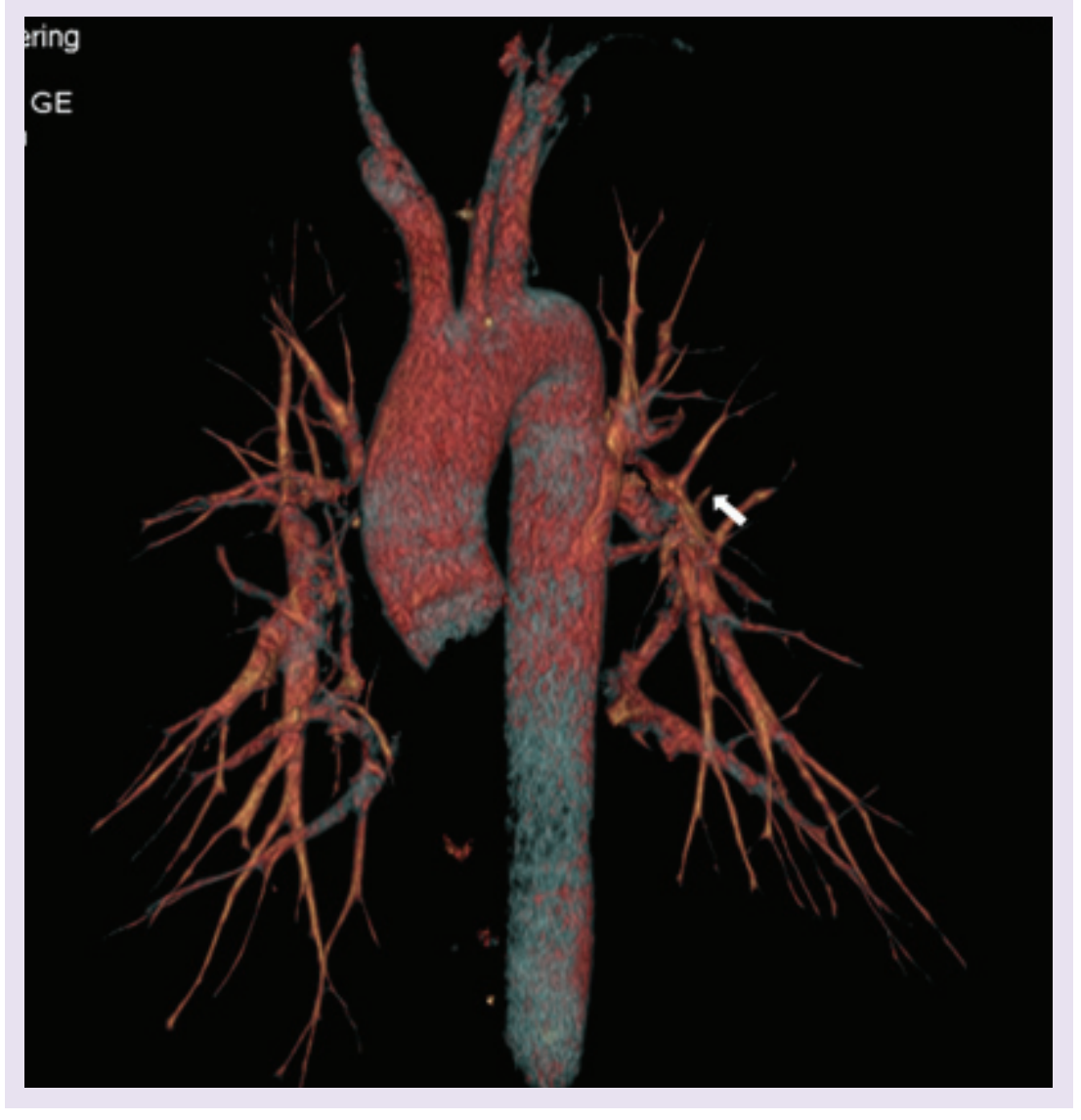

Figure 2.3D computed tomographic reconstruction in a 68-year-old man with right basal nodule. Vascular volume rendering reconstruction shows a mediastinal lingular artery (arrow).

3D CT could overcome this disadvantage; in that, it clearly shows structures and preoperative abnormalities [10]. Thus, 3D CT is useful for lung surgery and should be performed before surgery, if possible. Moreover, CT images of vascular structures, and in particular the possibility of maximum intensity projection, MPR and VR representation could update the existing classifications for a more effective and immediate consultation. The necessity of CT in the presurgical planning for the dual possibility of assessing either the lung parenchyma or vascular structures whose anatomic variants can modify the minimally invasive surgery is invaluable.

\section{Conclusion}

Knowledge of the frequency, extent of incomplete interlobar fissures and the presence of accessory fissures is part of basic anatomy. It is important for the thoracic surgeon to have access to optimal operative assessment and strategic planning in order to perform lobectomy and a safe 
surgery. In conclusion, identifying preoperative anatomic pulmonary changes with 3D CT, on the one hand, according to some surgeons with extensive experience in the field of VATS, is a disadvantageous instrument for the purpose of the procedure, considering the increased time consumption of 3D CT compared with traditional CT, except in selected clinical cases; on the other hand, considering that the average operation time of VATS lobectomy in patients with extended criteria is greater than in standards patients, it could be a valuable aid for surgeons who are less experienced in managing the possible complications described. This could provide them with a confirmed surgical approach and potentially safeguard the patient's health. Thoracic resection should be always preceded 3D CT reconstruction of the vascular tree and fissures using multiplanar data obtained with the CT test; it is possible to provide the interventional radiologist or surgeon, with essential, relevant help.

\section{Financial \& competing interests disclosure}

The authors have no relevant affiliations or financial involvement with any organization or entity with a financial interest in or financial conflict with the subject matter or materials discussed in the manuscript. This includes employment, consultancies, honoraria, stock ownership or options, expert testimony, grants or patents received or pending, or royalties.

No writing assistance was utilized in the production of this manuscript.

\section{Open access}

This work is licensed under the Creative Commons Attribution-NonCommercial 4.0 Unported License. To view a copy of this license, visit http://creativecommons.org/ licenses/by-nc-nd/4.0/

\section{References}

1 Taverne Y, Kleinrensink GJ, de Rooij P. Perioperative identification of an accessory fissure of the right lung. Case Rep. Pulmonol. 2015, 954769 (2015).

2 Ozmen CA, Nazaroglu H, Bayrak AH, Senturk S, Akay HO. Evaluation of interlobar and accessory pulmonary fissures on 64-row MDCT. Clin. Anat. 23(5), 552-558 (2010).

3 Esomonu UG, Taura MG, Modibbo MH, Egwu AO. Variation in the lobar pattern of the right and left lungs: a case report. Australas. Med. J. 6(10), 511-514 (2013).
4 Matsumoto K, Yamasaki N, Tsuchiya T et al. Three dimensional computed tomography for a mediastinal basal pulmonary artery. Ann. Thorac. Surg. 94(5), e115-e116 (2012).

5 Hayashi K, Aziz A, Ashizawa K, Hayashi H, Nagaoki K, Otsuji H. Radiographic and CT appearances of the major fissures. Radiographics 21(4), 861-874 (2001).

6 Galluzzo A, Genova C, Dioguardi S, Midiri M, Cajozzo M. Current role of computed tomography-guided transthoracic needle biopsy of metastatic lung lesions. Future Oncol. 11(Suppl. 2), 43-46 (2015).

7 Sofranik RM, Gross BH, Spizarny DL. Radiology of the pleural fissures. Clin. Imaging 16(4), 221-229 (1992).
8 Subotich D, Mandarich D, Milisavljevich M et al. Variations of pulmonary vessels: some practical implications for lung resections. Clin. Anat. 22, 698-705 (2009).

9 Bincy MG, Satheesha BN, Sapna M. Morphological variations of the lungs: a study conducted on Indian cadavers. Anat. Cell. Biol. 47, 253-258 (2014).

10 Yoshiaki M, Takase $\mathrm{K}$, Ichikawa $\mathrm{H}$ et al. Bronchial artery anatomy: preoperative $3 \mathrm{D}$ simulation with multidetector CT. Radiology 255(3), 934-943 (2010). 